\title{
Potential Interaction between Galectin-2 and MUC5AC in Mouse Gastric Mucus
}

\author{
Mayumi Tamura, ${ }^{a}$ Toru Tanaka, ${ }^{b}$ Norihiko Fujii, ${ }^{c}$ Takashi Tanikawa, ${ }^{a, b}$ Saori Oka, ${ }^{a}$ \\ Tomoharu Takeuchi, ${ }^{b}$ Tomomi Hatanaka, ${ }^{b, d}$ Seishi Kishimoto, ${ }^{c}$ and Yoichiro Arata ${ }^{*, a}$ \\ ${ }^{a}$ Faculty of Pharma-Science, Teikyo University; 2-11-1 Kaga, Itabashi-ku, Tokyo 173-8605, Japan: ${ }^{b}$ Faculty of \\ Pharmacy and Pharmaceutical Sciences, Josai University; 1-1 Keyakidai, Sakado, Saitama 350-0295, Japan: \\ ${ }^{c}$ Radioisotope Research Center, Teikyo University; 2-11-1 Kaga, Itabashi-ku, Tokyo 173-8605, Japan: and \\ ${ }^{d}$ Tokai University School of Medicine; 143 Shimokasuya, Isehara, Kanagawa 259-1193, Japan. \\ Received August 22, 2019; accepted October 24, 2019
}

Galectins are a group of animal lectins characterized by their specificity for $\beta$-galactosides. Of these, galectin-2 (Gal-2) is predominantly expressed in the gastrointestinal tract. In the current study, we used a mouse gastric mucous fraction to investigate whether Gal-2 is secreted from epithelial cells and identify its potential ligands in gastric mucus. Gal-2 was detected in the mouse gastric mucous fraction and could be eluted from it by the addition of lactose. Affinity chromatography using recombinant mouse galectin-2 (mGal-2)-immobilized adsorbent and subsequent LC-MS/MS identified MUC5AC, one of the major gastric mucin glycoproteins, as a potential ligand of mGal-2. Furthermore, MUC5AC was detected in the mouse gastric mucous fraction by Western blotting, and recombinant $\mathrm{mGal}-2$ was adsorbed to this fraction in a carbohydrate-dependent manner. These results suggested that Gal-2 and MUC5AC in mouse gastric mucus interact in a $\beta$-galactoside-dependent manner, resulting in a stronger barrier structure protecting the mucosal surface.

Key words galectin-2; MUC5AC; mucin; galectin; stomach

\section{INTRODUCTION}

Galectins comprise a type of animal lectins characterized by their specificity for $\beta$-galactosides and contain an evolutionarily conserved sequence motif in the carbohydrate-binding site. ${ }^{1-5)}$ Galectin-2 (Gal-2) was first identified in human $\left.{ }^{6}\right)$ and is highly expressed in gastric cells, predominantly in epithelial cells of the rat stomach.7) Mouse gastric surface mucous cells and mucous neck cells, which produce mucus, also show Gal-2 immunoreactivity ${ }^{8,9}$; however, no reaction was observed in parietal cells and chief cells. Furthermore, acute and chronic colitis in mice were alleviated upon Gal-2 overexpression. ${ }^{10)}$ Gal-2 mRNA has also been detected in the human stomach, ${ }^{11)}$ and reduced expression of human Gal-2 is associated with lymph node metastases in gastric cancer ${ }^{12)}$ and in Helicobacter-induced gastric cancer progression, ${ }^{13)}$ suggesting that Gal-2 plays a protective function in the gastrointestinal tract.

Gal-2 has a molecular weight of approximately $14 \mathrm{kDa}$ and is able to form homodimers in solution. ${ }^{14)}$ Thus, some of the functions of Gal-2 should be due to its potential ability to crosslink two molecules that possess the carbohydrate structure recognized by Gal-2. Recently, we reported that mouse Gal-2 (mGal-2) could interact with porcine mucin, an important component of gastric mucosa, in a $\beta$-galactosidedependent manner, resulting in an insoluble precipitate, potentially through the crosslinking of mucins and the formation of a lattice. This suggested that Gal-2 plays a role in the gastric mucosa by possibly strengthening the barrier structure through crosslinking the mucins on the mucosal surface. ${ }^{15)}$

Gastric mucins are large extracellular glycoproteins with high molecular weight. They are highly glycosylated, with approximately $80 \%$ of the mass of these molecules being derived from carbohydrates, and are the main component of mucus, which has viscous and elastic gel-like properties with many roles such as barrier to pathogens and gastric acids. ${ }^{16,17)}$ Cysteine-rich regions located at the amino and carboxyl terminus of the MUC polypeptide are involved in dimerization via disulfide bond formation; they can also form multimers by polymerization of the dimers. Currently, 21 mucin-type glycoproteins, which belong to the MUC gene family, have been identified in humans. Basically, mucins can be categorized into two groups, namely secreted mucins and tethered cell surface-associated mucins. ${ }^{17)}$ Among the secreted mucins, MUC2, MUC5AC, MUC5B, MUC6, and MUC19 form oligomers outside the epithelial cell layer via disulfide bonds and are gel-forming mucins. MUC7 and MUC8 are nonpolymeric secreted mucins. Among the gel-forming mucins, MUC5AC and MUC6 are the major secreted mucins in the stomach and are expressed by different gastric cellular populations. ${ }^{18-21)}$

Based on our previous observation, in the current study, we investigated whether mGal-2 is secreted in mouse gastric mucus and attempted to identify its potential ligand to gain mechanistic insights into its protective role in the gastrointestinal tract.

\section{MATERIALS AND METHODS}

Preparation of Recombinant mGal-2 The expression and purification of the recombinant wild-type mGal-2 was performed as previously described. ${ }^{22)}$

Animals Ten male ddY mice were purchased from Sankyo Labo Service Corporation, Inc. (Japan, Tokyo). All animal care procedures and experimental protocols were reviewed 
and approved by the Institutional Animal Care and Use Committee of Josai University (H29072).

Preparation of a Mouse Gastric Mucous Fraction The preparation of a mouse gastric mucous fraction was performed as previously described. ${ }^{23)}$

Isolation and Analysis of Possible mGal-2-Ligands in Gastric Mucus by LC-MS/MS The preparation of an mGal-2-immobilized column was performed as previously described. ${ }^{15)}$ Gastric mucous fractions from three mice were treated with phosphate buffered saline (PBS) and 5\% 2-mercaptoethanol overnight at $4{ }^{\circ} \mathrm{C}$ and then disrupted by sonication. The supernatant was separated by centrifugation at $17800 \times \boldsymbol{g}$ for $20 \mathrm{~min}$ at $4^{\circ} \mathrm{C}$ and applied to an mGal-2-immobilized column. The column was then washed with PBS, followed by elution with PBS containing $0.1 \mathrm{M}$ lactose. The collected fractions following lactose elution were subjected to acetone precipitation. The proteins in the precipitate were reduced by dithiothreitol and alkylated with iodoacetamide as described previously. ${ }^{24)}$ The $S$-carbamidomethylated proteins were digested with trypsin, using Trypsin Spin Columns (SIGMA, St. Louis, MO, U.S.A.) according to the manufacturer's instructions. The resulting peptides were sent to the Support Center for Advanced Medical Sciences, Tokushima University Graduate School of Biomedical Sciences for LC-MS/MS analysis. The results were filtered at $1 \%$ false discovery rate (FDR) using MASCOT.

Lactose Elution from the Mouse Gastric Mucous Fraction The pellet from the mouse gastric mucous fraction was obtained by centrifugation at $17800 \times \boldsymbol{g}$ for $20 \mathrm{~min}$ at $4^{\circ} \mathrm{C}$. The pellet was suspended in PBS containing $0.1 \mathrm{M}$ lactose and incubated overnight at $4^{\circ} \mathrm{C}$. After incubation, supernatant and precipitation fractions were separated by centrifugation, and both fractions were subjected to sodium dodecyl sulfatepolyacrylamide gel electrophoresis (SDS-PAGE) using sample buffer with or without $1 \%$ 2-mercaptoethanol.

Analysis of the Interaction between Recombinant mGal-2 and Stomach Mucus Recombinant mGal-2 $(10 \mathrm{ng} / \mu \mathrm{L})$ in PBS was added to the mouse gastric mucous pellet treated with $0.1 \mathrm{M}$ lactose and incubated overnight at $4^{\circ} \mathrm{C}$. $0.1 \mathrm{M}$ lactose or sucrose was added to the incubation mixture to test whether the interaction between $\mathrm{mGal}-2$ and the pellet could be inhibited.

SDS-PAGE, Western Blotting, and Lectin Blotting The samples were treated with SDS sample buffer containing $1 \%$ 2-mercaptoethanol and subjected to Western blotting using a rabbit polyclonal antibody raised against mGal-2 (CloudClone Corp., U.S.A.), or lectin blotting utilizing a biotinylated lectin from Glycine max (SBA) (J-Chemical, Inc., Japan) and biotinylated Ulex europaeus agglutinin I (UEA I) (Vector Laboratories, Inc., U.S.A.). For detection, HRP-labeled-anti rabbit immunoglobulin G (IgG) (Cell Signaling Technology, Inc., U.S.A.) and peroxidase-conjugated streptavidin (Thermo Scientific, U.S.A.) were used for Western blotting and lectin blotting, respectively.

For Western blot analysis of the mouse MUC5AC protein, the samples were treated with SDS sample buffer without 2-mercaptoethanol to avoid destruction of the epitope recognized by the MUC5AC monoclonal antibody (45M1) (GeneTex Inc., U.S.A.). For detection, HRP-labeled-anti-mouse IgG antibody (GE Healthcare UK Ltd., U.K.) was used.

LC-MS/MS For further analysis of the samples obtained from the mouse gastric mucous fraction, samples were denatured with $8 \mathrm{M}$ urea and $5 \mathrm{mM}$ dithiothreitol and then alkylated with $15 \mathrm{mM}$ iodoacetamide. Each sample was digested with trypsin/Lys-C mix (Promega, U.S.A.) and subjected to LC-MS/MS analysis using an Ultimate 3000 HPLC system coupled online to a Q Exactive orbitrap mass spectrometer (Thermo Fisher Scientific). The peptides were separated by RP-HPLC utilizing a C18 column (CAPCELL PAK C18 ACR, $1.0 \times 150 \mathrm{~mm}$, SHISEIDO, Japan) with a linear gradient of $0-50 \%$ acetonitrile in the presence of $0.1 \%$ formic acid at flow rate of $100 \mu \mathrm{L} / \mathrm{min}$.

The MS/MS spectra obtained were searched against the Mus musculus database downloaded from NCBI using the Sequest HT search engine incorporated in the Proteome Discoverer 2.2 (Thermo Fisher Scientific).

\section{RESULTS}

Isolation and Identification of an mGal-2 Ligand from the Mouse Gastric Mucous Fraction To find and identify potential mGal-2 ligands in the mouse gastric mucous fraction, affinity chromatography using an mGal-2-immobilized column was conducted. The adsorbed potential $\mathrm{mGal}-2$ ligands were subjected to protein identification by LC-MS/MS. When the results were filtered at $1 \%$ FDR using MASCOT, 351 proteins with scores higher than the peptide identity threshold were identified. Among them, the proteins with top 50 scores are listed in Supplementary Table 1. MUC5AC was at the top of the list with a score of 14422 , which was substantially higher than the second highest score for a protein, which was 3214 .

We have previously reported that $\mathrm{mGal}-2$ can interact with mucin from porcine stomach. ${ }^{15)}$ Since MUC5AC from mouse stomach was identified as a possible ligand for $\mathrm{mGal}-2$, and, furthermore, since mGal-2 and MUC5AC are both substantially expressed in gastric epithelial cells, ${ }^{8,21)}$ we decided to further investigate the interaction between $\mathrm{mGal}-2$ and MUC5AC.

mGal-2 Was Eluted with Lactose from the Mouse Gastric Mucous Fraction To test whether mGal-2 is associated with the mucus from mouse stomach, lactose was added to the gastric mucous fraction. After overnight incubation, soluble and insoluble fractions were separated by centrifugation. The whole mucous fraction (Whole), lactose-eluted fraction (Sup.), and remaining insoluble fraction (Ppt.) were subjected to Western blotting using anti-mGal-2 antibody. As shown in Fig. 1(A), bands with the size of an mGal-2 monomer (arrowhead in Fig. 1(A)) were detected with the mGal-2 antibody in the gastric mucous and lactose-eluted fractions, but not in the remaining precipitate. This result suggests that mGal-2 resides in the mucus and most of it was eluted with lactose, which might have competed with the interaction between mGal-2 and glycoprotein(s) containing carbohydrates with $\beta$-galactoside structures. The lactose-eluted fraction was analyzed by LC-MS/MS. Of the fourteen proteins identified, mGal-2 had the top sum Posterior Error Probabilities (PEP) score of 24.569 .

To test whether mucin exists in the remaining insoluble fraction following lactose elution, lectin blotting was conducted. Lectins used in this study were UEA-I, which recognizes glycoproteins and glycolipids containing $\alpha$-linked fucose residues on $O$-linked sugars, ${ }^{25-27)}$ and SBA, which binds to 
(A)

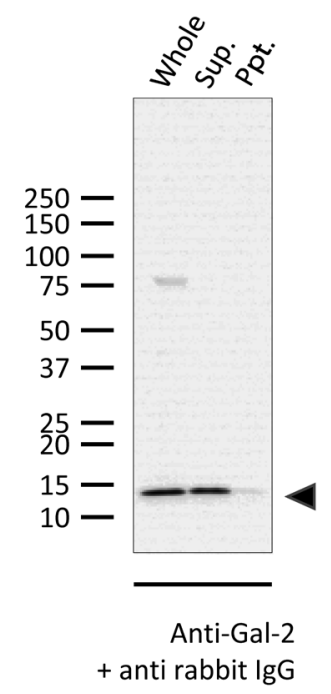

(B)

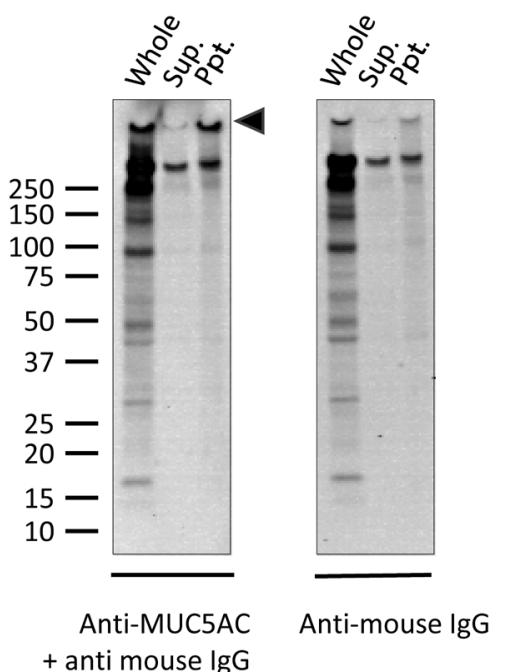

Fig. 1. mGal-2 Is Eluted by the Addition of Lactose to Stomach Mucus

The whole stomach mucous fraction (Whole), lactose-eluted fraction (Sup.), and remaining insoluble fraction (Ppt.) were subjected to Western blotting. (A) The three fractions were detected with anti-mGal-2 antibody. The black arrowhead shows the position of the mGal-2 monomer. (B) The three fractions were detected with antiMUC5AC antibody (left panel). Only the secondary antibody, anti-mouse IgG, was used as a control to identify non-specific mouse protein bands detected by anti-mouse IgG (right panel). The arrowhead in the left panel indicates the high molecular weight band detected by the anti-MUC5AC antibody, which was similar to the band of mouse MUC5AC.

the GalNAc $\alpha 1 \rightarrow 3$ Gal structure. ${ }^{28)}$ Smear bands at molecular weights higher than $150 \mathrm{kDa}$ were detected (data not shown). These results suggested that the remaining insoluble fraction after lactose elution contains glycoproteins with higher molecular weight including $O$-linked sugars, possibly mucins.

Since MUC5AC was identified as one of the main glycoproteins eluted from the mouse gastric mucous fraction that adsorbed to the mGal-2 affinity column, we investigated whether MUC5AC can be detected in the remaining insoluble fraction after lactose elution. The same three fractions analyzed in Fig. 1(A) were subjected to Western blotting using anti-MUC5AC. Several of the bands were detected even without utilizing the primary anti-MUC5AC antibody and only with the secondary anti-mouse $\mathrm{IgG}$, suggesting that these bands were non-specific (Fig. 1(B), right panel). When anti-MUC5AC was used, a clear high molecular weight band was detected (labeled with an arrowhead in Fig. 1(B), left panel), suggesting that MUC5AC exists in the mucous fraction from the mouse stomach and also remains in the insoluble fraction after lactose treatment. Furthermore, the remaining insoluble fraction was subjected to LC-MS/MS analysis, and MUC5AC was identified at the top of the list with the sum PEP score of 203.99, suggesting that MUC5AC indeed exists in this fraction as one of the main components. Although bands were detected at the top of the gel in both filters (Fig. 1(B)), the bands in the left panel (labeled with arrowhead) detected using anti-MUC5AC were substantially stronger than the ones detected in the right panel, suggesting that these include glycoproteins specifically recognized by the anti-MUC5AC antibody. Since the molecular weight(s) of the glycoproteins detected was very high, and the bands existed very close to the top of the gel, these bands could not be well separated from the other non-specific highmolecular weight glycoproteins detected by the secondary antibody (Fig. 1(B) right panel).

Added Recombinant mGal-2 Interacted with the Insoluble Fraction (Possibly via MUC5AC) in a CarbohydrateDependent Manner To test whether mGal-2 can interact

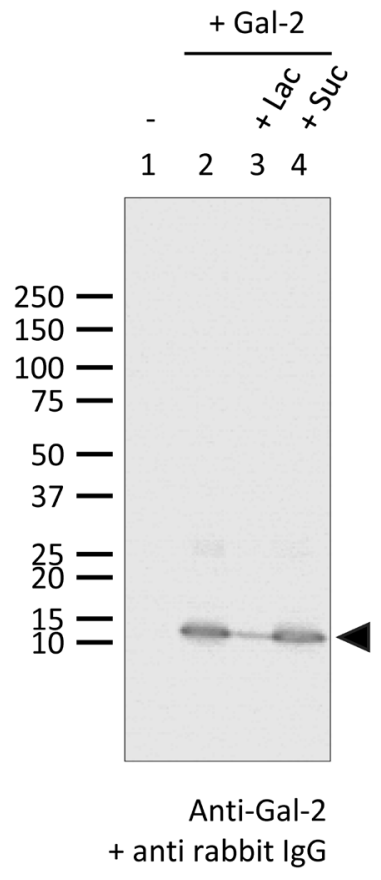

Fig. 2. Recombinant mGal-2 Binds in a $\beta$-Galactoside-Dependent Manner to the Remaining Residue Fraction of Mouse Gastric Mucus after Lactose Elution

The mouse gastric mucous fraction was treated with lactose, and the supernatant was removed. Recombinant $\mathrm{mGal}-2$ was added to the remaining insoluble residue in the presence of lactose or sucrose. Bound mGal-2 was detected by Western blotting using anti-mGal-2 antibody. Lane 1; no recombinant $\mathrm{mGal}-2$ added, lane 2; $\mathrm{mGal}-2$ added without the addition of lactose or sucrose, lane 3; mGal-2 added with $0.1 \mathrm{M}$ lactose, lane 4 ; mGal-2 added with $0.1 \mathrm{M}$ sucrose. (-); No mGal-2 was added. The black arrowhead indicates the position of the mGal-2 monomer.

with the insoluble fraction derived from mouse mucus after lactose treatment, recombinant $\mathrm{mGal}-2$ was added to this fraction. Added mGal-2 remained in the insoluble fraction and was detected by Western blotting (Fig. 2, lane 2). However, when lactose was also added to the mixture, the amount of mGal-2 detected was substantially smaller (Fig. 2, lane 3), 
suggesting that lactose inhibited the interaction between mGal-2 and MUC5AC in the insoluble fraction. This idea was further supported by the result that sucrose was not able to inhibit this interaction (Fig. 2, lane 4). These results suggested that $\mathrm{mGal}-2$ interacted with MUC5AC in the insoluble fraction in a $\beta$-galactoside-dependent manner.

\section{DISCUSSION}

Gal-2, which was first identified in human, ${ }^{6)}$ was found to be expressed predominantly in rat gastric epithelial cells ${ }^{7}$ and was detected in surface mucous cells and mucous neck cells in mice. ${ }^{8)}$ We previously reported that mGal-2 and porcine mucin form an insoluble strong complex, creating a matrix precipitate, which suggested that mGal-2 plays a role in strengthening the barrier structure in the gastric mucosa by crosslinking the mucins on the mucosal surface. ${ }^{15)}$ Galectin-3 forms a lattice barrier on the apical glycocalyx of ocular surface epithelial cells by interacting with the $O$-glycans of mucins. ${ }^{29)}$ In the current study, affinity chromatography and subsequent LC-MS/MS analysis indicated that one of the possible mGal-2 ligand(s) was MUC5AC. To further analyze the importance of mGal-2 and MUC5AC for the protection of the mucosal surface via potential crosslinking of MUC5AC by mGal-2, we initiated immunohistochemical studies to identify the localization of MUC5AC and mGal-2 in the mouse stomach. Furthermore, determining the localization of these proteins in stomach-related diseases such as gastric ulcer and cancer as well as differences during developmental stages and aging would help us understand the molecular mechanisms of barrier structure generation in the stomach as well as the etiology of gastric diseases.

As shown in Fig. 1, a substantial amount of mGal-2 was detected in the mouse gastric mucous fraction and was eluted with lactose from this fraction, suggesting that mGal-2 and MUC5AC form a complex in a $\beta$-galactoside dependent manner. Protein analysis in this fraction using LC-MS/MS identified mGal-2 and MUC5AC, along with the stomach mucous proteins, gastrokin-1 and trefoil factor 1, suggesting that both mGal-2 and MUC5AC indeed exist in the stomach mucus.

After elution of mGal-2 from the mucous fraction by lactose, MUC5AC remained in the insoluble fraction, along with other proteins such as gastrokin-2, gastrokin-1, mGal-2, and trefoil factor 1 . We postulate that MUC5AC remained in the insoluble fraction even after $\mathrm{mGal}-2$ elution, likely because mucins, including MUC5AC, were associated with each other by intermolecular disulfide bonds or hydrophobic interactions $^{16)}$ and possibly formed a high molecular weight multimer in the mucus. Moreover, mGal-2, which forms a homodimer, could strengthen this complex by crosslinking the MUC5AC molecules upon recognition of the sugar structure on the MUC5AC molecules. The analysis of the molecular properties of mucins is very difficult, since they have very high molecular mass and are enriched in glycans as can be seen in Fig. 1(B). However, future studies using supported molecular matrix electrophoresis devised specifically to analyze mucins ${ }^{30,31)}$ may lead to the molecular and functional characterization of MUC5AC, including the elucidation of its role as a ligand for $\mathrm{mGal}-2$.

MUC5AC, produced and secreted by the surface mucussecretor cells, is one of the major constituents of the gastric mucus. $^{32)}$ MUC5AC is extensively $O$-glycosylated ${ }^{33)}$ and is important for binding of Helicobacter pylori to the gastric mucosa in humans. ${ }^{34,35)}$ Although the $O$-glycans in mouse differ substantially from those of human MUC5AC, ${ }^{36)}$ MUC5AC from mouse also carries glycan structures such as blood group $\mathrm{H}$ antigen ${ }^{37)}$ that can be recognized by rat and human Gal-2 ${ }^{38,39)}$ and presumably by $\mathrm{mGal}-2$.

mGal-2 was identified in a screen of mouse gastric mucosal proteins that are uniquely sensitive to $S$-nitrosylation. ${ }^{40)}$ Although mGal-2 can lose its sugar-binding ability by oxidation, $S$-nitrosylation of $\mathrm{mGal}-2$ prevents this inactivation. ${ }^{22,41,42)}$ This suggests that although mGal-2 will lose its ability to interact and crosslink MUC5AC when oxidized, $S$-nitrosylation of this protein might prevent it from inactivation and maintain the strong barrier complex in the stomach, especially in an environment where secreted mucins are crosslinked by disulfide bonds. Thus, $S$-nitrosylation of $\mathrm{mGal}-2$ could be one of the important mechanisms for protecting the surface of the stomach when it is exposed to oxidative stress conditions that could lead to inactivation of the mGal-2 protein.

In summary, our study found that MUC5AC is one of the main ligands for $\mathrm{mGal}-2$ in the stomach. Our results suggest that crosslinking MUC5AC in a $\beta$-galactoside-dependent manner could result in strengthening of the barrier structure on the mucosal surface.

Acknowledgments This study was supported by the Support Center for Advanced Medical Sciences, Tokushima University Graduate School of Biomedical Sciences. The authors thank Ms. Ikuko Sagawa for technical assistance and helpful discussions. The authors also thank Ms. Sayaka Yoshida for technical assistance. This work was supported by JSPS KAKENHI Grant Number JP18K06634 (YA) and by research grants from the Teikyo University (YA and MT) and Josai University (MT, TT, TH, and YA).

Conflict of Interest The authors declare no conflict of interest.

Supplementary Materials The online version of this article contains supplementary materials.

\section{REFERENCES}

1) Kasai K, Hirabayashi J. Galectins: a family of animal lectins that decipher glycocodes. J. Biochem., 119, 1-8 (1996).

2) Cooper DN. Galectinomics: finding themes in complexity. Biochim. Biophys. Acta, 1572, 209-231 (2002).

3) Leffler H, Carlsson S, Hedlund M, Qian Y, Poirier F. Introduction to galectins. Glycoconj. J., 19, 433-440 (2002).

4) Boscher C, Dennis JW, Nabi IR. Glycosylation, galectins and cellular signaling. Curr. Opin. Cell Biol., 23, 383-392 (2011).

5) Di Lella S, Sundblad V, Cerliani JP, Guardia CM, Estrin DA, Vasta GR, Rabinovich GA. When galectins recognize glycans: from biochemistry to physiology and back again. Biochemistry, 50, 7842-7857 (2011).

6) Gitt MA, Massa SM, Leffler H, Barondes SH. Isolation and expression of a gene encoding L-14-II, a new human soluble lactosebinding lectin. J. Biol. Chem., 267, 10601-10606 (1992).

7) Oka T, Murakami S, Arata Y, Hirabayashi J, Kasai K, Wada Y, Futai M. Identification and cloning of rat galectin-2: expression is predominantly in epithelial cells of the stomach. Arch. Biochem. 
Biophys., 361, 195-201 (1999).

8) Nio-Kobayashi J, Takahashi-Iwanaga H, Iwanaga T. Immunohistochemical localization of six galectin subtypes in the mouse digestive tract. J. Histochem. Cytochem., 57, 41-50 (2009).

9) Nio-Kobayashi J. Tissue- and cell-specific localization of galectins, beta-galactose-binding animal lectins, and their potential functions in health and disease. Anat. Sci. Int., 92, 25-36 (2017).

10) Paclik D, Berndt U, Guzy C, Dankof A, Danese S, Holzloehner P, Rosewicz S, Wiedenmann B, Wittig BM, Dignass AU, Sturm A. Galectin-2 induces apoptosis of lamina propria $\mathrm{T}$ lymphocytes and ameliorates acute and chronic experimental colitis in mice. J. Mol. Med., 86, 1395-1406 (2008).

11) Saal I, Nagy N, Lensch M, Lohr M, Manning JC, Decaestecker C, Andre S, Kiss R, Salmon I, Gabius HJ. Human galectin-2: expression profiling by RT-PCR/immunohistochemistry and its introduction as a histochemical tool for ligand localization. Histol. Histopathol., 20, 1191-1208 (2005).

12) Jung JH, Kim HJ, Yeom J, Yoo C, Shin J, Yoo J, Kang CS, Lee C. Lowered expression of galectin-2 is associated with lymph node metastasis in gastric cancer. J. Gastroenterol., 47, 37-48 (2012).

13) Takaishi S, Wang TC. Gene expression profiling in a mouse model of Helicobacter-induced gastric cancer. Cancer Sci., 98, 284-293 (2007).

14) Lobsanov YD, Gitt MA, Leffler H, Barondes SH, Rini JM. X-ray crystal structure of the human dimeric $S$-Lac lectin, L-14-II, in complex with lactose at 2.9-A resolution. J. Biol. Chem., 268, 27034-27038 (1993).

15) Tamura $M$, Sato $D$, Nakajima $M$, Saito $M$, Sasaki T, Tanaka $T$, Hatanaka T, Takeuchi T, Arata Y. Identification of galectin-2-mucin interaction and possible formation of a high molecular weight lattice. Biol. Pharm. Bull., 40, 1789-1795 (2017).

16) Bansil R, Turner BS. Mucin structure, aggregation, physiological functions and biomedical applications. Curr. Opin. Colloid Interface Sci., 11, 164-170 (2006).

17) Wagner CE, Wheeler KM, Ribbeck K. Mucins and their role in shaping the functions of mucus barriers. Annu. Rev. Cell Dev. Biol., 34, 189-215 (2018).

18) De Bolós C, Garrido M, Real FX. MUC6 apomucin shows a distinct normal tissue distribution that correlates with Lewis antigen expression in the human stomach. Gastroenterology, 109, 723-734 (1995).

19) Ho SB, Roberton AM, Shekels LL, Lyftogt CT, Niehans GA, Toribara NW. Expression cloning of gastric mucin complementary DNA and localization of mucin gene expression. Gastroenterology, 109, 735-747 (1995)

20) Ho SB, Takamura K, Anway R, Shekels LL, Toribara NW, Ota $\mathrm{H}$. The adherent gastric mucous layer is composed of alternating layers of MUC5AC and MUC6 mucin proteins. Dig. Dis. Sci., 49, 1598-1606 (2004).

21) Nordman H, Davies JR, Lindell G, de Bolos C, Real F, Carlstedt I. Gastric MUC5AC and MUC6 are large oligomeric mucins that differ in size, glycosylation and tissue distribution. Biochem. J., 364, 191-200 (2002).

22) Tamura M, Saito M, Yamamoto $K$, Takeuchi T, Ohtake K, Tateno $\mathrm{H}$, Hirabayashi J, Kobayashi J, Arata Y. S-Nitrosylation of mouse galectin-2 prevents oxidative inactivation by hydrogen peroxide. Biochem. Biophys. Res. Commun., 457, 712-717 (2015).

23) Tani S, Okuda M, Morishige R, Tanaka T. Gastric mucin secretion from cultured rat epithelial cells. Biol. Pharm. Bull., 20, 482-485 (1997).

24) Takeuchi T, Nemoto-Sasaki Y, Arata Y, Kasai K. Galectin LEC-6 interacts with glycoprotein F57F4.4 to cooperatively regulate the growth of Caenorhabditis elegans. Biol. Pharm. Bull., 34, 11391142 (2011).

25) Kurimura Y, Tsuji Y, Yamamoto $K$, Kumagai H, Tochikura $T$. Efficient production and purification of extracellular 1,2-alpha-Lfucosidase of Bacillus sp. K40T. Biosci. Biotechnol. Biochem., 59,
589-594 (1995).

26) Pereira ME, Kisailus EC, Gruezo F, Kabat EA. Immunochemical studies on the combining site of the blood group H-specific lectin 1 from Ulex europeus seeds. Arch. Biochem. Biophys., 185, 108-115 (1978).

27) Pereira ME, Gruezo F, Kabat EA. Purification and characterization of lectin II from Ulex europaeus seeds and an immunochemical study of its combining site. Arch. Biochem. Biophys., 194, 511-525 (1979).

28) Pereira ME, Kabat EA, Sharon N. Immunochemical studies on the specificity of soybean agglutinin. Carbohydr. Res., 37, 89-102 (1974).

29) Argüeso P, Guzman-Aranguez A, Mantelli F, Cao Z, Ricciuto J, Panjwani N. Association of cell surface mucins with galectin-3 contributes to the ocular surface epithelial barrier. J. Biol. Chem., 284, 23037-23045 (2009).

30) Matsuno YK, Saito T, Gotoh M, Narimatsu H, Kameyama A. Supported molecular matrix electrophoresis: a new tool for characterization of glycoproteins. Anal. Chem., 81, 3816-3823 (2009).

31) Kameyama A, Yamakoshi K, Watanabe A. A rapid separation and characterization of mucins from mouse submandibular glands by supported molecular matrix electrophoresis. Biochim. Biophys. Acta. Proteins Proteomics, 1867, 76-81 (2019).

32) Reis CA, David L, Nielsen PA, Clausen H, Mirgorodskaya K, Roepstorff P, Sobrinho-Simoes M. Immunohistochemical study of MUC5AC expression in human gastric carcinomas using a novel monoclonal antibody. Int. J. Cancer, 74, 112-121 (1997).

33) Corfield AP. Mucins: a biologically relevant glycan barrier in mucosal protection. Biochim. Biophys. Acta, 1850, 236-252 (2015).

34) Magalhães A, Rossez Y, Robbe-Masselot C, Maes E, Gomes J, Shevtsova A, Bugaytsova J, Boren T, Reis CA. Muc5ac gastric mucin glycosylation is shaped by FUT2 activity and functionally impacts Helicobacter pylori binding. Sci. Rep., 6, 25575 (2016).

35) Duarte HO, Freitas D, Gomes C, Gomes J, Magalhaes A, Reis CA. Mucin-type $O$-glycosylation in gastric carcinogenesis. Biomolecules, 6, 33 (2016)

36) Kenny DT, Skoog EC, Linden SK, Struwe WB, Rudd PM, Karlsson NG. Presence of terminal $N$-acetylgalactosaminebetal- $4 N$-acetylglucosamine residues on $O$-linked oligosaccharides from gastric MUC5AC: involvement in Helicobacter pylori colonization? Glycobiology, 22, 1077-1085 (2012).

37) Holmén Larsson JM, Thomsson KA, Rodriguez-Pineiro AM, Karlsson H, Hansson GC. Studies of mucus in mouse stomach, small intestine, and colon. III. Gastrointestinal Muc5ac and Muc2 mucin $O$-glycan patterns reveal a regiospecific distribution. Am. J. Physiol. Gastrointest. Liver Physiol., 305, G357-G363 (2013).

38) Hirabayashi J, Hashidate T, Arata Y, Nishi N, Nakamura T, Hirashima M, Urashima T, Oka T, Futai M, Muller WE, Yagi F, Kasai K. Oligosaccharide specificity of galectins: a search by frontal affinity chromatography. Biochim. Biophys. Acta, 1572, 232-254 (2002).

39) Stowell SR, Arthur CM, Mehta P, Slanina KA, Blixt O, Leffler H, Smith DF, Cummings RD. Galectin-1, -2 , and -3 exhibit differential recognition of sialylated glycans and blood group antigens. J. Biol. Chem., 283, 10109-10123 (2008).

40) Ohtake K, Shimada N, Uchida H, Kobayashi J. Proteomic approach for identification of protein $S$-nitrosation in mouse gastric mucosa treated with $S$-nitrosoglutathione. J. Proteomics, 72, 750-760 (2009).

41) Tamura M, Sasai A, Ozawa R, Saito M, Yamamoto K, Takeuchi T, Ohtake K, Tateno H, Hirabayashi J, Kobayashi J, Arata Y. Identification of the cysteine residue responsible for oxidative inactivation of mouse galectin-2. J. Biochem., 160, 233-241 (2016).

42) Sakakura M, Tamura M, Fujii N, Takeuchi T, Hatanaka T, Kishimoto S, Arata Y, Takahashi H. Structural mechanisms for the $S$ nitrosylation-derived protection of mouse galectin-2 from oxidationinduced inactivation revealed by NMR. FEBS J., 285, 1129-1145 (2018). 